\title{
Modelamiento de la viscosidad dinámica de sustancias puras a partir de la teoría de entropía residual
}

\author{
Luis F. Cardona ${ }^{1^{*}}$, Jorge A. Velásquez ${ }^{2}$ y Luis A. Forero ${ }^{3}$ \\ (1) Facultad de Ingeniería Química, Escuela de Ingenierías, Grupo de Investigación en Pulpa y Papel, Universidad \\ Pontificia Bolivariana, Circular $1^{a}$ \# 70-01, Medellín-Colombia. (correo-e: luisfernando.cardonapa@upb.edu.co, \\ jorge.velasquezj@upb.edu.co, luis.foreroga@upb.edu.co). \\ *autor a quien debe ser dirigida la correspondencia
}

Recibido Nov. 12, 2020; Aceptado Ene. 13, 2021; Versión final Mar.15, 2021, Publicado Ago. 2021

\section{Resumen}

El propósito de este trabajo es desarrollar un modelo para la estimación de la viscosidad dinámica de fluidos Newtonianos en la curva de coexistencia líquido y vapor de sustancias no-polares y polares a partir de la teoría de entropía residual. Se utiliza una versión modificada de la ecuación de estado de Peng-Robinson. Se estudian 116 sustancias puras y el agua (67 sustancias para la correlación y 49 sustancias para la predicción). El modelo se generaliza para 12 familias orgánicas a partir de una función lineal en términos de la temperatura normal de ebullición. El intervalo de temperatura varía entre $104.81 \mathrm{~K}$ y $565.48 \mathrm{~K}$ y presión entre $0.01 \mathrm{kPa}$ y $3618.69 \mathrm{kPa}$. Las desviaciones absolutas promedio para la viscosidad obtenidas en la correlación y predicción son menores de $6.38 \%$ y $11.36 \%$, respectivamente. El modelo generalizado se valida frente a otros desarrollados en la literatura y los resultados obtenidos permiten concluir que el modelo propuesto es simple y proporciona resultados aceptables.

Palabras clave: viscosidad; ecuación de estado; sustancias puras; teoría de entropía residual; validación

\section{Modeling dynamic viscosity of pure substances based on entropy scaling theory}

\begin{abstract}
The present study aims to develop a model for dynamic viscosity estimation applied to Newtonian fluids in the liquid and vapor coexistence curve of non-polar and polar substances by applying residual entropy theory. A modified Peng-Robinson equation of state is used to calculate residual entropy. One-hundred and sixteen pure substances and water (67 substances for correlation and 49 substances for prediction) are studied. The model is generalized in terms of normal boiling temperature for 12 organic families using a linear function. The temperature range varies between $104.81 \mathrm{~K}$ and $565.48 \mathrm{~K}$ and the pressure ranges between $0.01 \mathrm{kPa}$ and $3618.69 \mathrm{kPa}$. The average absolute deviations for correlation and prediction are below $6.38 \%$ and $11.36 \%$, respectively. The generalized model is validated against others developed in the literature. Based on the results obtained, it is concluded that the proposed model is simple and provides acceptable results.
\end{abstract}




\section{INTRODUCCIÓN}

El conocimiento de la viscosidad en amplios intervalos de temperatura y presión es requerido en diferentes procesos industriales, que incluyen el diseño de equipos como separadores, torres de destilación y equipos de intercambio de calor (Márquez-Baños et al., 2016; Ortega et al., 2016). Una opción para estimar esta importante propiedad es el uso de datos experimentales (Páez et al., 2014), sin embargo, su obtención para diferentes tipos de fluidos en amplias condiciones de temperatura y presión es una tarea costosa y requiere mucho tiempo (Poling et al., 2001). Diferentes modelos han sido utilizados en la literatura para predecir la viscosidad entre los que se destacan los modelos teóricos, empíricos, semi-empíricos (Poling et al., 2001; Bonyadi et al., 2017; García et al., 2007) y los basados en redes neuronales e inteligencia artificial (Valderrama y Rojas, 2009). Los modelos teóricos se basan en simulaciones dinámicas moleculares y termodinámica estadística. Ejemplos de este tipo de modelos son los propuestos por Eyring y Chapman-Enskog (Poling et al., 2001). Sin embargo, altos porcentajes de error son obtenidos en la fase líquida (Bonyadi et al., 2017).

Los modelos empíricos son correlaciones basadas en la observación experimental y relaciona la viscosidad de líquidos o gases en términos de la temperatura y la presión (Poling et al., 2001). Algunos de estos se originan a partir del concepto de similitud geométrica entre dos diagramas termodinámicos como PvT y $T \mu P$. Ejemplos de este tipo de modelos son aquellos desarrollados por Bonyadi et al. (2017) y Cardona et al. (2019a). Recientemente, Valderrama et al. (2019) han utilizado este concepto para realizar predicciones de la viscosidad dinámica en líquidos iónicos con desviaciones inferiores de $5.60 \%$. Sin embargo, estos modelos tienen un gran número de parámetros y son aplicables para una familia orgánica en específica.

Por otro lado, las ecuaciones semi-empíricas son desarrolladas a partir de diferentes teorías como el principio de los estados correspondientes, la teoría de Eyring (Poling et al., 2001), la teoría de fricción (García et al., 2017), la teoría de entropía residual (Rosenfeld, 1977), entre otros (Satyro y Yarranton, 2010; Derevich, 2010). Estos modelos presentan una serie de parámetros los cuales se determinan utilizando datos experimentales. Además, requieren la estimación de propiedades termodinámicas como la entropía residual, la densidad, la entalpía residual, el factor de compresibilidad, entre otros. Las anteriores propiedades son usualmente estimadas a partir de ecuaciones de estado.

El último tipo de modelación de la viscosidad es aquella relacionada con redes neuronales e inteligencia artificial. Esta metodología se origina por la similitud de los sistemas biológicos que tienen la capacidad de aprendizaje y predicción (Valderrama et al., 2011; Faúndez y Campusano, 2018). El trabajo realizado por Valderrama et al. (2011) es uno de los más representativos que usa esta metodología aplicada a la viscosidad. Estos autores reportan desviaciones menores de $7.20 \%$ durante el entramiento de la red, mientras que durante el proceso de predicción se reporta desviaciones menores de $5.00 \%$. Una alternativa para modelar la viscosidad de sustancias no-polares y polares consiste en utilizar la teoría de entropía residual. Esta teoría fue inicialmente propuesta por Rosenfeld (1977) mientras realizaba un análisis de la relación entre la entropía residual y la viscosidad. Galliero et al. (2011) aplicaron la teoría de entropía residual para modelar la viscosidad del metano, butano, decano y argón con desviaciones promedio que varían entre $3.60 \%$ y $40.00 \%$.

Posteriormente, Novak (2011) realiza una modificación del modelo propuesto por Rosenfeld (1977) utilizando la relación de Chapman-Enskog (Poling et al., 2001) para 17 sustancias puras en su gran mayoría n-alcanos. Para el cálculo de la entropía residual, Novak (2011) utilizó la ecuación de estado PC-SAFT (Kontogeorgis y Folas, 2009). Las desviaciones obtenidas son inferiores de 6.70\%. Novak (2015) realiza una serie de modificaciones del modelo de viscosidad junto con la ecuación de estado PC-SAFT con el propósito de mejorar el desempeño del modelo en condiciones de alta temperatura y presión en la fase líquida para $\mathrm{n}$ alcanos. Las desviaciones obtenidas son inferiores de $6.60 \%$.

Lötgering-Lin y Gross (2015) desarrollan un modelo de viscosidad a partir de la teoría de entropía residual propuesta por Rosenfeld (1977) con cuatro parámetros ajustables. Los parámetros son generalizados utilizando técnicas de contribución de grupos junto con la ecuación de estado PCP-SAFT (Kontogeorgis y Folas, 2009). Lötgering-Lin y Gross (2015) reportan para 12 familias químicas que representan 110 sustancias puras desviaciones promedio que varían entre $4.81 \%$ y $30.83 \%$. No obstante, este modelo no realiza predicciones en compuestos isómeros y presenta altas desviaciones para n-alcoholes. Bell y Laesecke (2016) modelaron la viscosidad de refrigerantes utilizando el modelo de Rosenfeld (1977) junto con la ecuación reportada en la NIST-REFPROP. El modelo depende de un fluido de referencia que es seleccionado por tener la mayor cantidad de datos experimentales en la zona de saturación. Se consideró el R124 (1-cloro-1,2,2,2tetrafluoroetano) como fluido de referencia. Las desviaciones promedio para 10 refrigerantes son menores de $10.00 \%$. Recientemente, Bell (2019) realiza un análisis de la teoría de Rosenfeld (1977) junto con diferentes modificaciones que ha tenido este modelo durante los últimos años. El análisis demuestra la conexión entre la entropía residual y la viscosidad con bases moleculares, no obstante, altas desviaciones del $\pm 40 \%$ son obtenidas cuando se utiliza en refrigerantes y dióxido de carbono. 
En la literatura la teoría de entropía residual ha sido poco aplicada para estimar las viscosidades de sustancias polares. Además, los modelos generalizados de viscosidad son escasos. En este trabajo se utiliza la teoría de entropía residual desarrollada por Rosenfeld (1977) para modelar la viscosidad dinámica de fluidos Newtonianos no-polares y polares en la región de saturación. Esta región es de especial importancia en el diseño y optimización ya que permite realizar estimaciones preliminares de la viscosidad en equipos donde ocurren equilibrio de fases como separadores y torres de destilación. Para el cálculo de la entropía residual se utiliza una versión modificada de la ecuación de estado de Peng-Robinson (Valderrama, 2003) desarrollada por Forero y Velásquez (2019). El modelo semi-empírico de viscosidad se generaliza por familias orgánicas que son de interés en la industria petroquímica y de disolventes (Poling et al., 2001). Finalmente se compara con otros desarrollados en la literatura.

\section{OTROS ANTECEDENTES}

Hay una serie de antecedentes adicionales que es necesario detallar para documentar en mejor forma este trabajo: modelo de viscosidad y modelo de ecuación de estado.

\section{Modelo de viscosidad}

Una alternativa para modelar la viscosidad es la utilización del concepto de entropía residual. El modelo semiempírico desarrollado por Rosenfeld (1977) es utilizado en este trabajo y su expresión matemática es:

$$
\mu^{r}=\frac{\mu}{\mu_{0}}=A \cdot \exp \left(\frac{B \cdot S^{r e s}}{R}\right)
$$

En la Ecuación (1), $A$ y $B$ son parámetros empíricos del modelo, $R$ es la constante de los gases ideales $\left(R=8.314472 \mathrm{~kJ} \cdot \mathrm{kmol}^{-1} \cdot \mathrm{K}^{-1}\right), \mu^{r}$ es la viscosidad residual estimada como $\mu^{r}=\mu / \mu_{0}$, donde $\mu$ y $\mu_{0}$ tienen unidades de $\mu \mathrm{P}$ (micropoise, $1 \mu \mathrm{P}=10^{-7} \mathrm{~Pa} \cdot \mathrm{s}$ ). $\mu_{0}$ representa la viscosidad de gas diluido (Poling et al., 2001). Además, $S^{\text {res }}$ es la entropía residual estimada como: $S^{r e s}(T, P)=S_{(T, P)}-S^{G l}(T, P)$ y $S^{G l}$ es la entropía en la condición de gas ideal. En particular, para este trabajo se estima la $S^{r e s}$ a partir de una modificación de la ecuación de estado de Peng-Robinson (Valderrama, 2003) propuesto por Forero y Velásquez (2019). Para el cálculo de la viscosidad de gas diluido se utiliza la ecuación de Chung et al. (Poling et al., 2001). Este modelo es desarrollado a partir de la teoría cinética de los gases y se ha utilizado en diferentes aplicaciones académicas e industriales (Poling et al., 2001). La expresión matemática que representa el gas diluido se muestra a continuación.

$$
\mu_{0}=\frac{40.785 \sqrt{M_{w} T}}{v_{c}^{2 / 3} \Omega^{*}} F_{c}
$$

En la Ecuación (2), $\mu_{0}$ es la viscosidad de gas diluido en micropoise $(\mu P), M_{w}$ es el peso molecular en $\mathrm{g} \cdot \mathrm{mol}^{-1}, v_{c}$ es el volumen crítico en $\mathrm{cm}^{3} \cdot \mathrm{mol}^{-1} \mathrm{y} \Omega^{*}$ corresponde a la integral de colisión reducida que se calcula a partir de la aproximación propuesta por Neufeld et al. (Poling et al., 2001), así:

$$
\begin{aligned}
\Omega^{*}=\frac{1.16145}{T^{* 0.14874}}+ & \frac{0.52487}{\exp \left(0.77320 T^{*}\right)}+\frac{2.16178}{\exp \left(2.43787 T^{*}\right)}-6.435 \\
& \times 10^{-4} T^{* 0.14874} \sin \left(18.0323 T^{*-0.76830}-7.27371\right)
\end{aligned}
$$

En la Ecuación (3), $T=1$.2593. $T_{r}$ y $T_{r}$ es la temperatura reducida calculada como $T_{r}=T / T_{c}, F_{c}$ es un factor empírico introducido por Chung et al. (Poling et al., 2001) con el propósito de mejorar las predicciones en sustancias polares y se calcula de acuerdo con la Ecuación (4):

$$
F_{c}=1-0.2756 \omega+0.059035 \cdot u_{r}^{4}+\kappa
$$

En la Ecuación (4), $\omega$ es el factor acéntrico (adimensional), $\kappa$ es un parámetro empírico que tiene en cuenta la formación de puentes de hidrógeno y se calcula como: $\kappa=0.0682+4.704 \cdot[n u ́ m e r o ~ d e ~ g r u p o s ~ O H-] / M_{w}$. El término $u_{r}$ se estima como $u_{r}=131.3 \cdot u \cdot\left(v_{c} \cdot T_{c}\right)^{-1 / 2}$, donde $T_{c}$ es la temperatura crítica en Kelvin, $v_{c}$ es el volumen crítico en $\mathrm{cm}^{3} \cdot \mathrm{mol}^{-1} \mathrm{y} u$ es el momento dipolar (Debye). En particular, el valor de $\kappa$ y $u_{r}$ para hidrocarburos es despreciable. Las propiedades críticas $\left(T_{c}, P_{c}\right)$, el factor acéntrico $(\omega)$, el momento dipolar $(u)$ y el volumen crítico $\left(v_{c}\right)$ son obtenidos a partir de los valores recomendados como aceptados por la base de datos de la 
DIPPR (Daubert, 1998). El modelo de viscosidad es consistente en unidades, donde: $S^{\text {res }}$ está en $\mathrm{kJ} \cdot \mathrm{kmol}^{-1} \cdot \mathrm{K}^{-1}$, $T$ en Kelvin, $P$ en $\mathrm{kPa}, \mu$ y $\mu_{0}$ en $\mu \mathrm{P}$ (micropoise), $A$ y $B$ son adimensionales.

\section{Modelo de ecuación de estado}

Las ecuaciones cúbicas del tipo van der Waals son las más utilizadas en la industria de procesos ya que permiten realizar predicciones de equilibrio de fases, simular y diseñar diferentes equipos de ingeniería (Valderrama, 2003). Recientemente, Forero y Velásquez (2019) desarrollan una versión modificada de la ecuación de Peng-Robinson (Valderrama, 2003) y ha sido generalizada. Para 259 sustancias puras (81 no polares y 178 polares) genera desviaciones menores del 5.00\% cuando se utiliza para estimar la presión de vapor, volumen molar y capacidades caloríficas. El modelo se comparó con las ecuaciones de PengRobinson, Peng-Robinson-Stryjek-Vera y Patel-Teja (Kleiman et al., 2002, Valderrama, 2003) y los resultados muestran las excelentes capacidades predictivas del modelo desarrollado por Forero y Velásquez (2019). Además, se ha utilizado de manera satisfactoria en la modelación y diseño de procesos industriales (Benabithe et al., 2019). Por estas razones se ha seleccionado la versión modificada de la ecuación de estado de Peng-Robinson para describir la entropía residual de las sustancias puras. En la Tabla 1 se muestra un resumen del modelo de ecuación de estado (EoS).

Tabla 1: Ecuaciones matemáticas del modelo de EoS desarrollado por Forero y Velásquez, 2019.

\begin{tabular}{|c|c|}
\hline Descripción & Ecuaciones matemáticas \\
\hline Forma explícita de la EoS & $P=\frac{R T}{v+c-b}-\frac{a}{v^{2}+2 v(c+b)+c^{2}+2 c b-b^{2}}$ \\
\hline Parámetros de la EoS & $\begin{aligned} & a_{i}=a_{c i} \alpha_{i}=0.45723553 \frac{\left(R T_{C i}\right)^{2}}{P_{C i}} \exp \left[H_{1 i}\left(1-\left(\frac{T}{T_{C i}}\right)^{H_{2 i}}\right)\right], \quad b_{i}=0.07779607 \frac{R T_{C i}}{P_{C i}} \\
c_{i}= & {\left[v_{P R}-v_{E x p}\right]_{T_{r}^{L}=0.7} }\end{aligned}$ \\
\hline Ecuaciones generalizadas & $\begin{array}{l}\omega_{i}=-1-\left.\log \frac{P^{s a t}}{P_{c}}\right|_{T_{r}=0.7}, \quad \chi_{i}=\left.\log \frac{P^{s a t}}{P_{c}}\right|_{T_{r}=0.6}+1.7 \omega_{i}+1.552 \\
H_{2 i}=\frac{\gamma_{c i}}{\beta_{c i}-1}+\beta_{c i} ; H_{1 i}=\frac{\beta_{c i}-1}{H_{2 i}} \\
\beta_{c i}=1.34695+1.49228 \omega_{i}+1.4252 \chi_{i}-0.12039 \omega_{i}^{2}+2.8166 \chi_{i}^{2}+0.312707 \omega_{i} \chi_{i} \\
\gamma_{c i}=-0.37592-1.44144 \omega_{i}+6.20445 \chi_{i}-0.67473 \omega_{i}^{2}+15.586 \chi_{i}^{2}-0.70582 \omega_{i} \chi_{i}\end{array}$ \\
\hline $\begin{array}{l}\text { Ecuación de la entropía } \\
\text { residual }\end{array}$ & $\Delta S^{r e s}=\left(s-s^{G I}\right)=R \ln \left[Z\left(1-\frac{b}{v}\right)\right]-\frac{1}{2 \sqrt{2} b R}\left(\frac{\partial a}{\partial T}\right)_{v} \ln \left[\frac{v+c+b-\sqrt{2} b}{v+c+b+\sqrt{2} b}\right]$ \\
\hline
\end{tabular}

Los parámetros $a$ y $b$ (ver Tabla 1, fila 3) del modelo de ecuación de estado se refieren al término atractivo y repulsivo, respectivamente. Por otro lado, el parámetro de traslación en el volumen, $c$, tiene la capacidad de mejorar el desempeño del modelo para describir las densidades de líquidos. El modelo reportado en la Tabla 1 se encuentra generalizado a partir del factor acéntrico ( $\omega$, adimensional) y el factor polar de Halm-Stiel, $\chi$ (Forero y Velásquez, 2019). En las ecuaciones reportadas en la Tabla 1 se debe observar lo siguiente: 1) el subíndice $i$ representa la sustancia a evaluar; 2 ) la función alfa ( $\alpha_{i}$, ver Tabla 1 fila 3 ) es aquella propuesta por Heyen (Valderrama, 2003) donde $H_{1 i}$ y $H_{2 i}$ corresponden a los parámetros ajustables; 3 ) el parámetro de traslación, $c_{i}$, se calcula como la diferencia entre el volumen molar no trasladado y el volumen experimental estimado a partir de los valores reportados como aceptados por la base de datos de la DIPPR (Daubert, 1998) a $T_{r}^{L}=0.7\left(T_{r}=T / T_{c}\right.$, donde $T$ es la temperatura y $T_{c}$ es la temperatura crítica en Kelvin); 4) el parámetro de translación se encuentra reportado en la literatura para un gran número de sustancias (Forero y Velásquez, 2019); 5) el factor de compresibilidad ( $Z$, ver Tabla 1 fila 5) se estima como $Z=P \cdot v \cdot R^{-1} \cdot T^{-1}$; 6 ) el modelo es consistente en unidades, donde $T$ es la temperatura en Kelvin, $P$ es la presión en $\mathrm{kPa}, \omega, \chi, Z$ son adimensionales, $v$ es el volumen molar en $\mathrm{m}^{3} \cdot \mathrm{kmol}^{-1}, S^{\text {res }}$ (entropía residual) en $\mathrm{kJ} \cdot \mathrm{kmol}^{-1} \cdot \mathrm{K}^{-1}$.

\section{METODOLOGÍA}

El modelo semi-empírico de entropía residual presenta dos parámetros ajustables $(A$ y $B)$ los cuales deben ser determinados utilizando datos experimentales de viscosidad. Para todas las sustancias puras se emplean los datos reportados como aceptados por la base de datos de la DIPPR (Daubert, 1998). Esta base de datos es ampliamente utilizada en la obtención de diferentes tipos de modelos que incluyen ecuaciones de estado (Forero y Velásquez, 2019) y propiedades de transporte (Cardona et al., 2019a). En la Tabla 2 se reportan 
los intervalos de temperatura, presión y viscosidad para cada familia orgánica analizada en este trabajo (Tabla 2, columnas 5, 6 y 7). Estos intervalos incluyen condiciones de alta y baja temperatura y presión a excepción del punto crítico de cada sustancia. Los datos comprendidos en la región cercana al punto crítico no son tenidos en cuenta en el proceso de correlación, ya que de acuerdo con lo reportado en la literatura los modelos de ecuación de estado del tipo van der Waals o SAFT (Lötgering-Lin y Gross, 2015) disminuyen su precisión en la vecindad del punto crítico, por lo que será un tema para desarrollar en trabajos futuros. Además, en la Tabla 2 se reportan las sustancias utilizadas durante el proceso de correlación y predicción (columnas 3 y 4). La selección de las sustancias en la correlación se realiza a partir de las series homólogas de cada familia orgánica. Además, las sustancias predichas pueden ser los consecutivos de las series homólogas u otras sustancias que pertenezcan a la familia orgánica. Finalmente, en la columna 8 de la misma tabla se tiene la incertidumbre experimental reportada por la base de datos de la DIPPR (Daubert, 1998). La DIPPR asigna los valores de incertidumbre para cada sustancia según el tipo de dato y la información proporcionada por los autores en sus trabajos originales como los métodos utilizados y la incertidumbre en la toma de los datos (Bloxham et al., 2020).

Para cada sustancia se utilizan 22 datos de saturación, distribuidos en 11 de líquido y 11 de vapor e igualmente espaciados. La literatura reporta entre 10 y 24 datos experimentales para el ajuste del modelo (Cardona et al., 2019a; García et al., 2017). En este trabajo se toma un valor de 22 datos (11 de líquido y 11 de vapor) para la determinación de las constantes que se encuentra dentro del rango utilizado para el ajuste de modelos de transporte (viscosidad y conductividad térmica). Es importante aclarar que la entropía residual que aparece en el modelo de viscosidad es estimada a partir de la modificación de la ecuación de estado de Peng-Robinson desarrollada por Forero y Velásquez (2019). En total, se evalúan 116 sustancias distribuidas entre 64 no-polares y 52 polares, que corresponden a 12 familias orgánicas, donde 67 sustancias son utilizadas en el proceso de correlación y 49 en el proceso de predicción. El número total de datos son de 2552 distribuidos en 1486 durante el proceso de correlación y 1066 en la predicción.

Luego de recolectar los datos experimentales y calcular para cada valor de temperatura y presión la entropía residual se utiliza un proceso de optimización a partir el método del Gradiente Reducido Generalizado (Cardona et al., 2019b) incorporado en Solver de MS Excel, junto con la función objetivo a minimizar dada por la siguiente ecuación matemática:

$$
\text { F.O. }=A A D \mu(\vec{X})=\frac{1}{N S} \sum_{j=1}^{N S}\left[\frac{1}{N D_{j}} \sum_{i=1}^{N D_{j}}\left|\frac{\mu_{\exp _{i}}-\mu_{\text {cal }}(\vec{X})_{i}}{\mu_{\exp _{i}}}\right|\right]_{j}
$$

Donde $\boldsymbol{X}=\{A, B\}$. En la Ecuación (5), $A A D \mu(X)$ es la suma total de la desviación absoluta relativa de la viscosidad de la fase líquida o vapor, $N S$ es el número de sustancias, $N D j$ es el número de datos de viscosidad de cada sustancia, $\mu_{\text {exp }}$ y $\mu_{c a l}$ representan las viscosidades experimental y calculada y $X$ es el vector de parámetros: $A_{i}, B_{i}$ que depende de cada sustancia ( $I$ y de la fase (líquido o vapor). Además, se estima la desviación relativa $(A D \mu)$, el valor máximo de la desviación absoluta relativa $(M D \mu)$ y el valor mínimo de la desviación absoluta relativa (mín. $A A D \mu$ ), así:

$$
\begin{aligned}
& A D \mu=\frac{100}{N D} \sum_{i=1}^{N D}\left(\frac{\mu_{c a l}-\mu_{\text {exp }}}{\mu_{\text {exp }}}\right) \\
& M D \mu=\text { valor máximo de } 100 \cdot\left|\frac{\mu_{c a l}-\mu_{\text {exp }}}{\mu_{\text {exp }}}\right| \\
& \text { mín. } A A D \mu=\text { valor mínimo de } 100 \cdot\left|\frac{\mu_{\text {cal }}-\mu_{\text {exp }}}{\mu_{\text {exp }}}\right|
\end{aligned}
$$

Las Ecuaciones 5 - 8 representan variables estadísticas de decisión para indicar la bondad y robustez de un modelo (Cardona et al., 2019b). Con el conjunto de parámetros óptimos se grafican en términos de la temperatura normal de ebullición. La temperatura de ebullición es un descriptor molecular utilizado en la literatura para generalizar modelos de viscosidad, conductividad térmica y tensión superficial (Cardona et al., 2019a; Poling et al., 2001). La temperatura de ebullición es obtenida a partir de los datos reportados como aceptados por la base de datos de la DIPPR (Daubert, 1998). Se toman como referencia los n-alcanos y nalcoholes. La Figura 1 ilustra el comportamiento de los parámetros óptimos en términos de la temperatura de ebullición. Se identifica una clara tendencia lineal de los parámetros en la fase líquida, mientras que un comportamiento constante de los parámetros en la fase vapor. El coeficiente de determinación es mayor de 0.8448 . 
Tabla 2: Intervalos de temperatura, presión, viscosidad e incertidumbre experimental $\pm \% \Delta \mu$ ( $N=$ No. total de sustancias).

\begin{tabular}{|c|c|c|c|c|c|c|c|c|}
\hline \multirow{2}{*}{ No. } & \multirow{2}{*}{$\begin{array}{l}\text { Familia } \\
\text { orgánica }\end{array}$} & \multicolumn{2}{|c|}{ Sustancias analizadas } & \multicolumn{3}{|c|}{$\begin{array}{l}\text { Intervalos de temperatura, presión y } \\
\text { viscosidad }\end{array}$} & \multirow{2}{*}{ $\pm \% \Delta \mu$} & \multirow{2}{*}{$N$} \\
\hline & & $\begin{array}{l}\text { Proceso de } \\
\text { Correlación }\end{array}$ & $\begin{array}{l}\text { Proceso de } \\
\text { Predicción }\end{array}$ & $\Delta T(\mathrm{~K})$ & $\Delta P(\mathrm{kPa})$ & $\Delta \mu(\mu \mathrm{P})$ & & \\
\hline 1 & $\begin{array}{l}\text { alcanos } \\
\text { ramificados }\end{array}$ & $\begin{array}{l}\text { 2-metilpropano, } \\
\text { 2-metilbutano, } \\
\text { 2-metilpentano, } \\
\text { 3-metilpentano, } \\
\text { 3-etil pentano }\end{array}$ & $\begin{array}{l}\text { 2,2-dimetilpropano, } \\
\text { 2,2-dimetilbutano, } \\
\text { 2,4-dimetil } \\
\text { pentano, 2,2,4- } \\
\text { trimetilpentano, } \\
\text { 2,3,4- } \\
\text { trimetilpentano }\end{array}$ & $240-373$ & $17.84-136.6$ & $60.4-4497.8$ & $5-10$ & 10 \\
\hline 2 & aldehídos & $\begin{array}{l}\text { metanal, } \\
\text { acetaldehído, } \\
\text { propanal, butanal }\end{array}$ & 2-metilpropanal & 204-322 & $1.7-66.3$ & $62.3-9316.0$ & $5-10$ & 5 \\
\hline 3 & aminas & $\begin{array}{l}\text { metanamina, } \\
\text { etanamina } \\
\text { 1-propanamina, } \\
\text { 1-butanamina }\end{array}$ & $\begin{array}{l}\text { 2-propanamina, } \\
\text { etilendiamina, } \\
\text { dimetil amina, } \\
\text { n-etiletanamina } \\
\text { 2-metilpropan-1- } \\
\text { amina }\end{array}$ & $223-367$ & 3.04-100.6 & $62.1-13356.9$ & $5-10$ & 9 \\
\hline 4 & cetonas & $\begin{array}{l}\text { 2-propanona, } \\
\text { 2-butanona, } \\
\text { 2-pentanona, } \\
\text { 3-pentanona, } \\
\text { 3-hexanona, } \\
\text { 2-heptanona }\end{array}$ & $\begin{array}{l}\text { 3-metilbutan-2- } \\
\text { ona, 4- } \\
\text { metilpentan-2-ona, } \\
\text { 5-nonanona }\end{array}$ & $254-384$ & $0.35-40.8$ & $56.2-8159.9$ & $3-5$ & 9 \\
\hline 5 & aromáticos & $\begin{array}{l}\text { benceno, } \\
\text { metilbenceno, } \\
\text { 1,2-dimetilbenceno, } \\
\text { 1,3-dimetilbenceno, } \\
\text { 1,4-dimetilbenceno }\end{array}$ & $\begin{array}{l}\text { etenilbenceno, } \\
\text { etilbenceno, } \\
\text { n-butilbenceno }\end{array}$ & $314-422$ & $6.51-123.0$ & $71-4893.1$ & $3-5$ & 8 \\
\hline 6 & cicloalcanos & $\begin{array}{l}\text { ciclopropano, } \\
\text { ciclobutano, } \\
\text { ciclopentano }\end{array}$ & ciclohexano & $210-348$ & $10.56-155.6$ & $62.5-9570.4$ & $1-25$ & 4 \\
\hline 7 & esteres & $\begin{array}{l}\text { metilbutanoato, } \\
\text { etil butanoato, } \\
\text { propil butanoato }\end{array}$ & dietil butanodioato & $255-397$ & $0.01-21.9$ & $54.8-20257.8$ & $5-10$ & 4 \\
\hline 8 & éteres & $\begin{array}{l}\text { metoximetano, } \\
\text { dimetoximetano, } \\
\text { 1-metoxipropano, } \\
\text { etoxietano, } \\
\text { 1-metoxibutano, } \\
\text { 1-metoxipentano }\end{array}$ & $\begin{array}{l}\text { 1.2-dimetoxietano, } \\
\text { 2-metoxi-2- } \\
\text { metilpropano, } \\
\text { 2-metoxipropano }\end{array}$ & $180-327$ & $0.21-69.5$ & $51.9-9826.0$ & $5-10$ & 9 \\
\hline 9 & alcanos & metano a decano & $\begin{array}{l}\text { undecano a } \\
\text { eicosano }\end{array}$ & $104-565$ & $0.01-318.6$ & $40.7-11085.1$ & $3-10$ & 20 \\
\hline 10 & alquenos & eteno a 1-deceno & $\begin{array}{l}\text { 1-undeceno a } 1 \text { - } \\
\text { eicoseno }\end{array}$ & $149-485$ & $0.06-163.2$ & $49.8-8912.5$ & $5-10$ & 19 \\
\hline 11 & alquinos & acetileno, 1-propino & 1-pentino & $200-360$ & $99.2-726.6$ & $67.6-1930.7$ & $5-25$ & 3 \\
\hline \multirow[t]{2}{*}{12} & alcoholes & metanol a 1-decanol & $\begin{array}{l}\text { 1-undecanol a } \\
\text { 1-pentadecanol }\end{array}$ & $230-410$ & $0.01-6.7$ & $47.7-103169.1$ & $5-10$ & 15 \\
\hline & agua & & & $291-517$ & $2.0-3618.6$ & $95.2-10687.0$ & $1-3$ & 1 \\
\hline
\end{tabular}



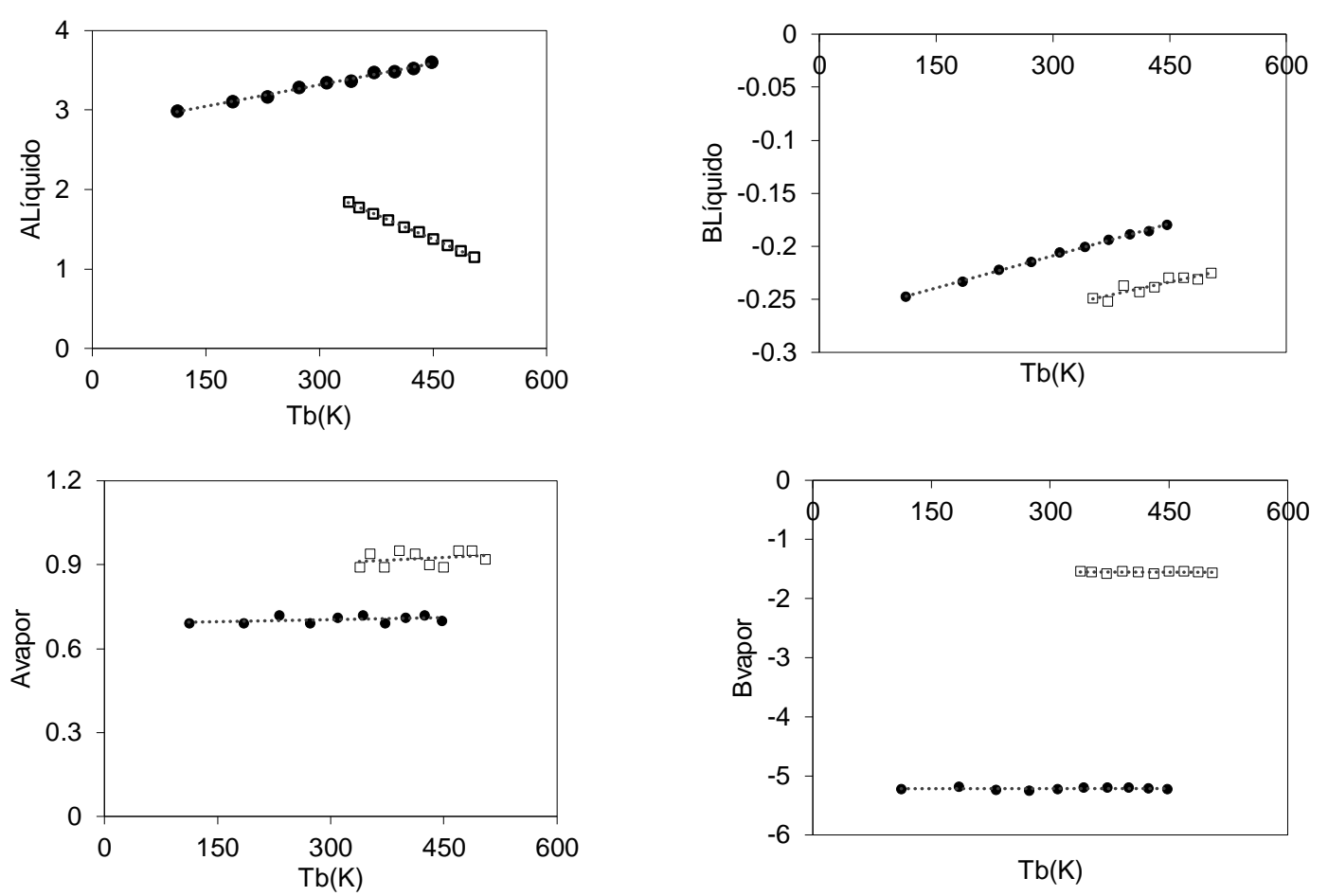

Figura 1: Variación de los parámetros $A$ y $B$ en términos de la temperatura normal de ebullición. $\square: \mathrm{n}$-alcoholes, $\bullet: \mathrm{n}$ alcanos. La línea punteada indica la tendencia lineal.

Posteriormente, para cada familia orgánica reportada en la Tabla 2 se estiman los valores de las pendientes y los interceptos, tanto para la fase líquida como vapor utilizando la función objetivo a minimizar dada por la Ecuación 5 y el método del Gradiente Reducido Generalizado incorporado en Solver de MS Excel (Cardona et al., 2019b). La Tabla 3 muestra para cada familia orgánica la generalización de cada parámetro en términos de la temperatura de ebullición. Es importante aclarar, que la expresión matemática utilizada durante el proceso de generalización es del tipo lineal: $Z_{i}=$ Pendiente $\times T_{b}+$ Intercepto, donde $Z_{i}=A, B$ y $T_{b}$ es la temperatura normal de ebullición en Kelvin. En especial para la fase vapor, la pendiente presenta un valor de cero, tal como se muestra en la Figura 1. En particular, el metano, el metanol y el agua presentan un comportamiento diferente al resto de las sustancias (Lötgering-Lin y Gross, 2015), por estos motivos los parámetros son estimados como un grupo aparte (ver Tabla 3). Es importante aclarar que los parámetros del modelo son empíricos y varían dentro del conjunto de los números reales que incluyen valores positivos, negativos y el cero (Lötgering-Lin y Gross, 2015).

\section{RESULTADOS Y DISCUSIÓN}

Para cada una de las familias orgánicas reportadas en la Tabla 2 junto con las expresiones de generalización de los parámetros $A$ y $B$ (Tabla 3) se estiman las desviaciones (ver Ecuaciones 5 - 8) y los resultados se muestran en las Tablas 4 y 5 . En la Tabla 4 se observa los resultados obtenidos durante el proceso de correlación mientras que en la Tabla 5 los de predicción. Es importante aclarar que los resultados corresponden a condiciones de saturación. Trabajos posteriores son necesarios para evaluar la capacidad de correlación y predicción en la región de una sola fase.

En total, 1486 datos reportados por la base de datos de la DIPPR (Daubert, 1998) son utilizados durante el proceso de correlación (Tabla 4, columna 2). El 14.54\% de los datos (216 de 1486 datos) presentan desviaciones absolutas superiores a $10 \%$, no obstante, ningún valor tiene desviaciones superiores de $30.00 \%$. Las familias orgánicas que presentan las mayores desviaciones son los cicloalcanos (11.43\%) y los alquinos (11.78\%). Para estos fluidos las incertidumbres experimentales se encuentren entre el $5.00 \%$ y $25.00 \%$, respectivamente. Por otro lado, 1066 datos son utilizados durante el proceso de predicción. En la Tabla 5, el $23.36 \%$ de los datos (249 de 1066 datos) presentan desviaciones absolutas mayores a $10.00 \%$, mientras que el $2.72 \%$ de los datos (29 de 1066 datos) sus desviaciones son superiores a $30.00 \%$. El número de sustancias junto con los datos analizados son representativos cuando se comparan con otros trabajos de la literatura (Derevich, 2010; Bonyadi. y Rostami, 2017; Cardona et al., 2019a).

En total, 2552 datos son utilizados en ambos procesos (correlación y predicción), donde el $18.22 \%$ de los datos (465 de 2552 datos) presenta desviaciones superiores a $10 \%$ y el $1.14 \%$ de los datos (29 de 2552 datos) sus desviaciones son superiores a $30.00 \%$. En general, en la correlación la $A A D \mu$ es menor de $6.38 \%$ 
(mín. $A A D \mu=2.82 \cdot 10^{-10 \%}$ y $M D \mu=26.44 \%$ ), mientras que en la predicción es menor de $11.36 \%$ (mín. $A A D \mu=6.84 \cdot 10^{-9} \%$ y $M D \mu=37.61 \%$ ). Estos resultados muestran las adecuadas capacidades del modelo para describir la viscosidad con desviaciones promedio que se encuentran dentro de la incertidumbre experimental.

Tabla 3. Tabla de generalización de los parámetros del modelo de entropía residual.

\begin{tabular}{|c|c|c|c|c|}
\hline \multirow{2}{*}{ Familia orgánica } & \multirow{2}{*}{ Parámetros } & \multicolumn{2}{|c|}{ Fase líquida } & \multirow{2}{*}{$\begin{array}{l}\text { Fase vapor } \\
\text { Intercepto }\end{array}$} \\
\hline & & Pendiente & Intercepto & \\
\hline \multirow{2}{*}{ alcanos ramificados } & $A$ & -0.0069 & 8.5283 & 1.0601 \\
\hline & B & $-8.4865 \cdot 10^{-5}$ & -0.15528 & 0.4772 \\
\hline \multirow{2}{*}{ aldehídos } & $A$ & 0.00870 & -1.23544 & 0.8256 \\
\hline & B & 0.00026 & -0.33519 & 0.0596 \\
\hline \multirow{2}{*}{ aminas } & $A$ & -0.01126 & 5.35983 & 0.9642 \\
\hline & $\mathrm{B}$ & -0.00075 & -0.01429 & 0.2782 \\
\hline \multirow{2}{*}{ cetonas } & $A$ & -0.004775 & 4.834525 & 0.8894 \\
\hline & $\mathrm{B}$ & -0.000106 & -0.153542 & 1.0976 \\
\hline \multirow{2}{*}{ aromáticos } & $A$ & 0.012519 & -2.352797 & 0.88810 \\
\hline & $\mathrm{B}$ & 0.000757 & -0.523808 & -2.49400 \\
\hline \multirow{2}{*}{ ciclo alcanos } & A & 0.02907 & -4.10737 & 0.9022 \\
\hline & $\mathrm{B}$ & 0.00009 & -0.23166 & -1.0010 \\
\hline \multirow{2}{*}{ esteres } & $A$ & -0.01961 & 10.06737 & 0.89450 \\
\hline & $\mathrm{B}$ & -0.00055 & 0.00146 & -0.93681 \\
\hline \multirow{2}{*}{ éteres } & $A$ & 0.03032 & -6.24295 & 0.8743 \\
\hline & $A$ & 0.00043 & -0.32122 & 1.2378 \\
\hline \multirow{2}{*}{ n-alcanos } & $A$ & 0.00100 & 2.59575 & 0.9000 \\
\hline & B & 0.00018 & -0.27974 & -2.3019 \\
\hline \multirow{2}{*}{ n-alquenos } & $A$ & 0.00196 & 2.72457 & 1.00000 \\
\hline & B & 0.00017 & -0.26063 & -1.38682 \\
\hline \multirow{2}{*}{ n-alquinos } & $A$ & -0.01982 & 9.30526 & 0.93781 \\
\hline & B & 0.00018 & -0.20666 & -0.17183 \\
\hline \multirow{2}{*}{ n-alcoholes } & A & -0.00367 & 3.91673 & 0.9611 \\
\hline & B & 0.00017 & -0.29348 & -2.1924 \\
\hline \multirow{2}{*}{ metano } & $A$ & 0.00000 & 1.8849 & 0.9000 \\
\hline & $B$ & 0.00000 & -0.2955 & -2.3019 \\
\hline \multirow{2}{*}{ metanol } & $A$ & 0.00000 & 1.8472 & 0.9611 \\
\hline & $B$ & 0.00000 & -0.2263 & -2.1924 \\
\hline \multirow{2}{*}{ agua } & $A$ & 0.00000 & 0.46616 & 0.73767 \\
\hline & $\mathrm{B}$ & 0.00000 & -0.27437 & -0.17120 \\
\hline
\end{tabular}

En la Figura 2 se muestran los valores calculados y experimentales de la viscosidad durante el proceso de correlación y predicción. En general los datos calculados presentan un buen comportamiento con relación a los datos reportados por la base de datos de la DIPPR tanto en la correlación como predicción con un coeficiente de determinación superior de 0.9551. En las Figuras 3 y 4 se ilustran las capacidades de correlación y predicción del modelo.

La Figura 3 corresponde a las viscosidades calculadas durante el proceso de correlación mientras que la Figura 4 muestra las viscosidades predichas. La Figura $3 a$ muestra los siguientes n-alcanos utilizados en el proceso de correlación, $\mathbf{m}$ : propano, $\square$ : butano, $\bullet$ : pentano, ○: hexano, $\mathbf{\Delta}$ : heptano, $\Delta$ : octano, x: nonano, +: decano, mientras que en la Figura $3 b$ ilustra los siguientes n-alquenos, a: propeno, $\square$ : 1-buteno, $\bullet$ : 1-penteno, $\boldsymbol{\Delta}$ : 1-hepteno, $\Delta$ : 1-octeno, x: 1-noneno, +: 1-deceno. Por otro lado, la Figura 4a muestra los siguientes nalcanos predichos: $\bullet$ : undecano, ०: dodecano, $\mathbf{m}$ : tridecano, $\square$ : tetradecano, $\bullet$ : pentadecano, mientras que la Figura 4b ilustra los siguientes $n$-alquenos predichos, $\bullet$ : 1-undeceno, $\mathbf{m}$ : 1-trideceno, $\bullet$ : 1-pentadeceno, $\Delta$ : 1 octadeceno.

Como se observan en estas Figuras, las viscosidades calculadas representan de manera adecuada las viscosidades reportadas en la base de datos de la DIPPR (Daubert, 1998). Es importante anotar que para la fase líquida a medida que aumenta la viscosidad se incrementa las desviaciones experimentales ya que se acerca a la condición crítica (Lötgering-Lin y Gross, 2015) y de acuerdo con la literatura en la vecindad del punto crítico se incrementa la incertidumbre en la medición lo que genera dispersión en el modelo. 
Tabla 4: Desviaciones relativas y absolutas durante el proceso de correlación.

\begin{tabular}{|l|c|c|c|c|c|c|c|c|c|c|c|}
\hline \multirow{2}{*}{$\begin{array}{c}\text { Familia } \\
\text { química }\end{array}$} & $N$ & \multicolumn{4}{|c|}{ Fase líquida } & \multicolumn{3}{c|}{ Fase vapor } & \multicolumn{2}{c|}{ Rangos de desviación } \\
\cline { 2 - 12 } & & $A D \mu$ & $A D D \mu$ & $\begin{array}{c}\text { mín } \\
A A D \mu\end{array}$ & $M D \mu$ & $A D \mu$ & $A D D \mu$ & $\begin{array}{c}\text { No. } \\
A A D \mu\end{array}$ & $\begin{array}{c}\text { No. Datos } \\
\text { Con } \\
A A D \mu>30\end{array}$ \\
\hline $\begin{array}{l}\text { alcanos } \\
\text { ramificados }\end{array}$ & 110 & -0.92 & 7.41 & $2.47 \cdot 10^{-1}$ & 20.95 & 2.03 & 2.29 & $1.33 \cdot 10^{-6}$ & 3.32 & 13 & 0 \\
\hline aldehídos & 88 & 1.02 & 3.29 & $8.66 \cdot 10^{-5}$ & 12.28 & 1.56 & 4.46 & $2.82 \cdot 10^{-10}$ & 10.99 & 13 & 0 \\
\hline aminas & 88 & -3.88 & 4.56 & $5.54 \cdot 10^{-2}$ & 15.91 & 0.57 & 1.15 & $9.31 \cdot 10^{-2}$ & 2.05 & 4 & 0 \\
\hline cetonas & 132 & 0.23 & 3.23 & $4.85 \cdot 10^{-5}$ & 12.28 & 2.67 & 4.18 & $1.20 \cdot 10^{-6}$ & 13.62 & 14 & 0 \\
\hline aromáticos & 110 & -3.12 & 6.71 & $4.82 \cdot 10^{-2}$ & 17.21 & -7.7 & 7.72 & $6.24 \cdot 10^{-1}$ & 12.66 & 28 & 0 \\
\hline cicloalcanos & 66 & -2.31 & 11.43 & $6.70 \cdot 10^{-1}$ & 20.24 & -8.11 & 8.11 & 3.18 & 11.79 & 27 & 0 \\
\hline esteres & 66 & -1.64 & 7.32 & $1.20 \cdot 10^{-1}$ & 26.44 & 0.55 & 0.64 & $4.83 \cdot 10^{-3}$ & 2.21 & 15 & 0 \\
\hline éteres & 132 & -3.82 & 6.74 & $2.06 \cdot 10^{-4}$ & 23.3 & 7.15 & 8.07 & $7.98 \cdot 10^{-6}$ & 27.34 & 37 & 0 \\
\hline alcanos & 220 & 0.64 & 2.27 & $5.53 \cdot 10^{-6}$ & 10.97 & -2.16 & 5.11 & $5.70 \cdot 10^{-2}$ & 15.94 & 10 & 0 \\
\hline alquenos & 198 & 3.14 & 4.17 & $1.60 \cdot 10^{-1}$ & 10.98 & 0.74 & 2.95 & $1.31 \cdot 10^{-3}$ & 10.35 & 12 & 0 \\
\hline alquinos & 44 & 1.22 & 11.78 & 5.29 & 17.24 & -5.05 & 5.05 & 3.21 & 6.99 & 15 & 0 \\
\hline alcoholes & 220 & -1.35 & 7.00 & $1.05 \cdot 10^{-3}$ & 20.26 & -1.71 & 2.2 & $1.70 \cdot 10^{-2}$ & 10.09 & 27 & 0 \\
\hline agua & 12 & -1.39 & 7.09 & $2.04 \cdot 10^{-5}$ & 17.95 & 0.31 & 0.62 & $9.09 \cdot 10^{-3}$ & 1.41 & 1 & 0 \\
\hline General & 1486 & -0.94 & 6.38 & $5.53 \cdot 10^{-6}$ & 26.44 & -0.70 & 4.04 & $2.82 \cdot 10^{-10}$ & 27.34 & 216 & 0 \\
\hline
\end{tabular}

Tabla 5: Desviaciones relativas y absolutas durante el proceso de predicción.

\begin{tabular}{|c|c|c|c|c|c|c|c|c|c|c|c|}
\hline \multirow[b]{2}{*}{ Familia química } & \multicolumn{5}{|c|}{ Fase líquida } & \multicolumn{4}{|c|}{ Fase vapor } & \multicolumn{2}{|c|}{ Rangos de desviación } \\
\hline & $N$ & $A D \mu$ & $A D D \mu$ & $\begin{array}{c}\text { mín } \\
A A D \mu\end{array}$ & $M D \mu$ & $A D \mu$ & $A D D \mu$ & $\begin{array}{c}\text { mín } \\
A A D \mu\end{array}$ & $M D \mu$ & $\begin{array}{l}\text { No. Datos } \\
\text { con } \\
A A D \mu>10\end{array}$ & $\begin{array}{c}\text { No. Datos } \\
\text { con } \\
A A D \mu>30\end{array}$ \\
\hline $\begin{array}{l}\text { alcanos } \\
\text { ramificados }\end{array}$ & 110 & -13.42 & 15.4 & $2.49 \cdot 10^{-6}$ & 35.68 & -0.6 & 2.06 & $6.84 \cdot 10^{-9}$ & 5.41 & 33 & 7 \\
\hline aldehídos & 22 & -11.17 & 11.17 & 3.05 & 17.8 & -5.70 & 5.70 & 5.52 & 5.76 & 7 & 0 \\
\hline aminas & 110 & -1.99 & 15.93 & $7.59 \cdot 10^{-6}$ & 35.95 & -3.53 & 8.56 & $1.31 \cdot 10^{-4}$ & 15.1 & 66 & 12 \\
\hline cetonas & 66 & 0.30 & 6.65 & $1.19 \cdot 10^{-2}$ & 13.52 & -1.1 & 2.02 & $2.35 \cdot 10^{-2}$ & 4.77 & 8 & 0 \\
\hline aromáticos & 66 & -7.39 & 8.52 & $3.65 \cdot 10^{-2}$ & 23.29 & -8.67 & 8.67 & $4.81 \cdot 10^{-1}$ & 14.9 & 23 & 0 \\
\hline cicloalcanos & 22 & -19.64 & 19.64 & 9.71 & 32.56 & -10.22 & 10.22 & 7.25 & 12.44 & 16 & 1 \\
\hline esteres & 22 & -11.41 & 11.41 & $1.33 \cdot 10^{-1}$ & 21.6 & -9.26 & 9.26 & 8.56 & 9.51 & 6 & 0 \\
\hline éteres & 66 & -0.78 & 18.07 & 1.26 & 37.61 & -3.1 & 3.1 & $9.54 \cdot 10^{-1}$ & 7.4 & 27 & 5 \\
\hline alcanos & 220 & 1.38 & 2.56 & $2.60 \cdot 10^{-2}$ & 10.65 & -3.98 & 4.28 & $3.37 \cdot 10^{-3}$ & 8.46 & 1 & 0 \\
\hline alquenos & 220 & -6.17 & 6.44 & $2.20 \cdot 10^{-1}$ & 16.62 & -4.66 & 4.66 & $7.81 \cdot 10^{-1}$ & 7.99 & 17 & 0 \\
\hline alquinos & 22 & -9.17 & 9.17 & 3.53 & 14.96 & -5.09 & 5.09 & 3.82 & 6.08 & 5 & 0 \\
\hline alcoholes & 110 & 1.26 & 16.08 & $5.78 \cdot 10^{-1}$ & 36.17 & 1.44 & 3.31 & 1.62 & 6.86 & 39 & 4 \\
\hline agua & 10 & -5.1 & 6.59 & $3.23 \cdot 10^{-3}$ & 14.88 & -0.22 & 0.57 & $2.09 \cdot 10^{-7}$ & 0.88 & 1 & 0 \\
\hline General & 1066 & -6.41 & 11.36 & $2.49 \cdot 10^{-6}$ & 37.61 & -4.21 & 5.19 & $6.84 \cdot 10^{-9}$ & 15.10 & 249 & 29 \\
\hline
\end{tabular}



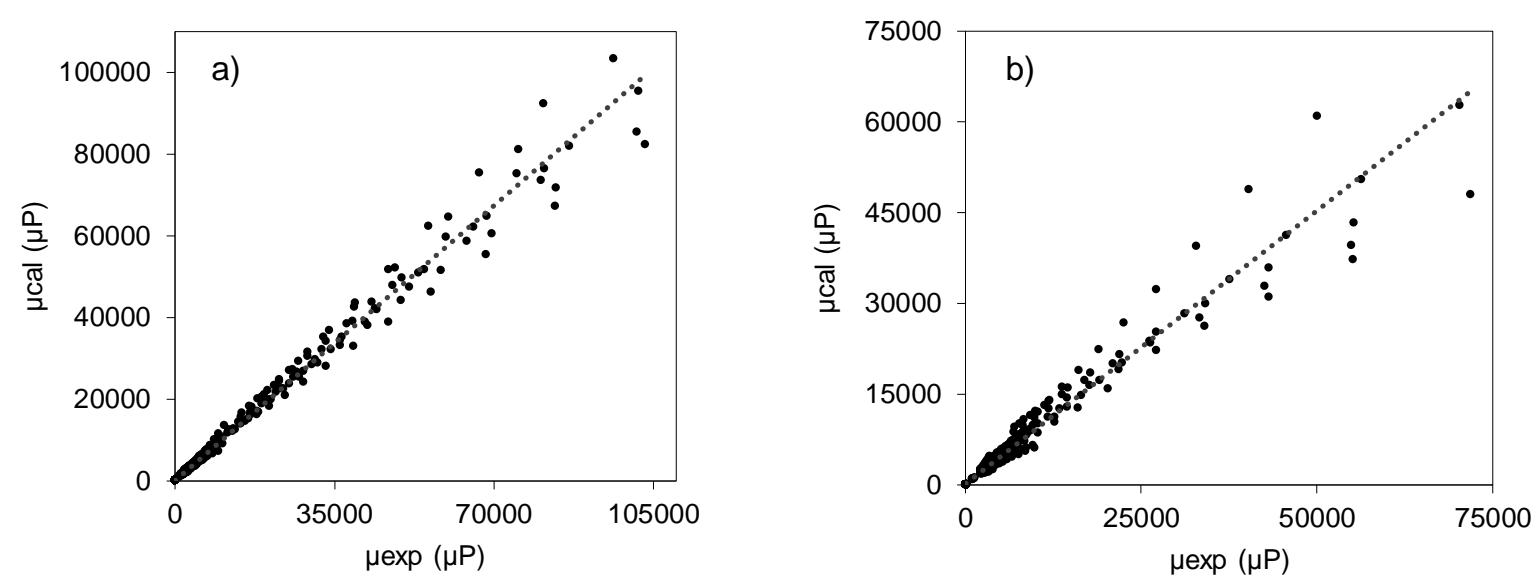

Figura 2: Viscosidades calculadas vs. experimentales usando el modelo de entropía residual generalizado. a) Proceso de correlación y b) Proceso de predicción.
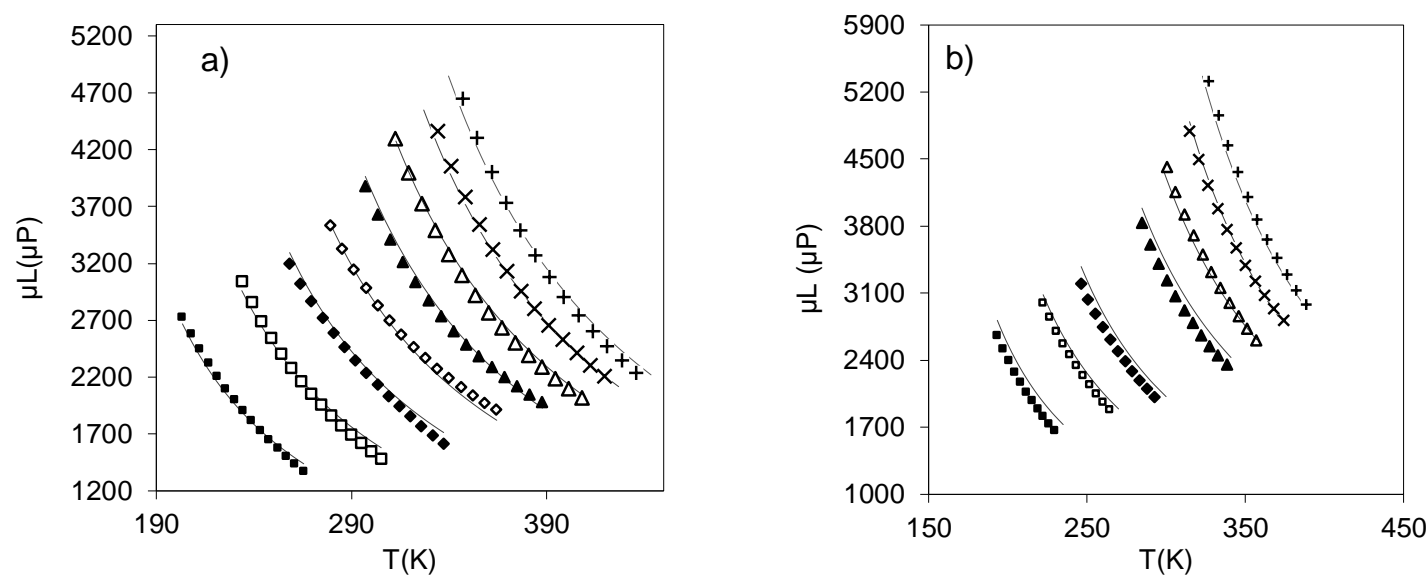

Figura 3: Comparación entre las viscosidades de líquido calculadas y experimentales durante el proceso de correlación para (a) n-alcanos, b) alquenos. La línea sólida corresponde al modelo generalizado.
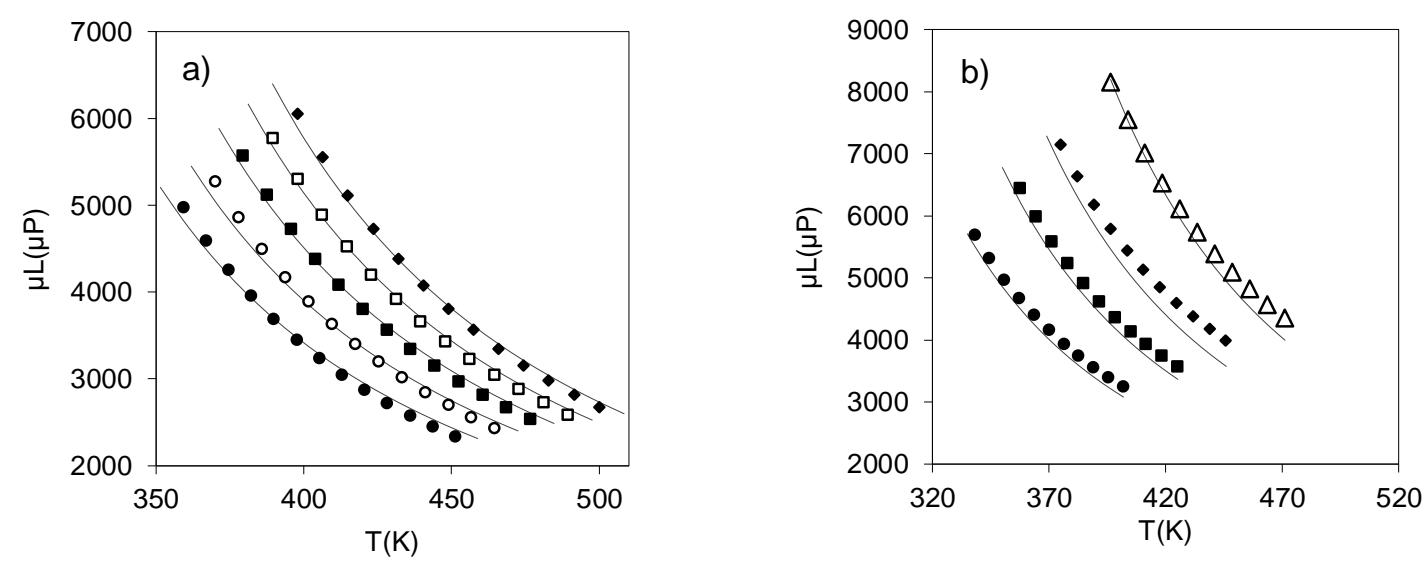

Figura 4: Comparación entre las viscosidades de líquido calculadas y experimentales durante el proceso de predicción para (a) n-alcanos, (b) alquenos. La línea sólida corresponde al modelo generalizado.

\section{DISCUSION FINAL}

La aplicación de la teoría de entropía residual principalmente se ha realizado en la estimación de las viscosidades de hidrocarburos, en particular para n-alcanos (Novak, 2015; Bonyadi y Rostami, 2017; Cardona et al., 2019a). Si la comparación se realiza con el uso de similares sustancias, el modelo propuesto por Lötgering-Lin y Gross (2015) para fase líquida cumple con estas características. Estos autores, utilizan la técnica de contribución de grupos para generalizar los cuatro parámetros del modelo de viscosidad junto con 
la ecuación de estado PCP-SAFT para el cálculo de la entropía residual. Sin embargo, los autores no reportan los intervalos de temperatura y presión utilizados durante el proceso de correlación y predicción y los valores máximos de desviación, por lo que el análisis se encuentra limitado. Las desviaciones absolutas relativas promedio $(A A D \mu)$ obtenidas por Lötgering-Lin y Gross (2015) son menores de $6.00 \%$, donde los aldehídos, los $\mathrm{n}$-alcoholes y los alcanos ramificados tienen valores de $A A D \mu$ entre $10.00 \%$ y $25.00 \%$. Para este tipo de sustancias en este trabajo se obtienen valores de $A A D \mu$ durante el proceso de correlación para los aldehídos de $3.29 \%$ (mín. $A A D \mu=8.66 \cdot 10^{-5} \%$ y $M D \mu=12.28 \%$ ), los n-alcoholes de $7.00 \%$ (mín. $A A D \mu=1.05 \cdot 10^{-3} \%$ y $M D \mu=20.26 \%$ ) y los alcanos ramificados de $7.41 \%$ (mín. $A A D \mu=2.47 \cdot 10^{-1} \%$ y $M D \mu=20.95 \%$ ).

Finalmente, el modelo generalizado se compara con otros reportados en la literatura tal como se muestra en la Tabla 6. Los modelos descritos en esta Tabla se originan por diferentes teorías de viscosidad como el transporte de momentum, entropía residual, concepto de similitud geométrica y expansión de un fluido (Tabla 6, columna 3). La gran mayoría de estos modelos son utilizados para sustancias no-polares, especialmente para n-alcanos (Tabla 6, columna 5). Además, los modelos 1 y 4 (Tabla 6 , columna 1 ) requieren del cálculo de la densidad, el modelo 3 de la entropía residual, y el modelo 1 de la entalpía residual y el factor de compresibilidad. Frente al número de constantes asociadas a los modelos reportados en esta Tabla (columna 4) varían entre 4 (Derevich) y 16 (Satyro y Yarranton), en comparación con el modelo desarrollado en este trabajo que cuenta con 6 constantes por familia orgánica. Además, estos modelos tienen valores máximos de desviación absoluta superiores a los obtenidos en este trabajo o no se reportan estos valores. Las $A A D \mu$ (desviación absoluta relativa promedio) de los modelos reportados en la Tabla 6 son menores de 7.79\%, mientras que en este trabajo las $A A D \mu$ son menores de $5.81 \%$ durante el proceso de correlación y $8.25 \%$ durante el proceso de predicción. Los valores de $A A D \mu$ y $M D \mu$ reportados en la Tabla 6 (última columna) son obtenidos a partir del promedio entre la fase líquida y vapor. Los anteriores resultados, muestran las adecuadas capacidades correlativas y predictivas del modelo desarrollado en este trabajo cuando son comparadas con otros modelos.

Tabla 6: Comparación y validación de diferentes modelos de viscosidad asociados a una ecuación de estado.

\begin{tabular}{|c|c|c|c|c|c|c|c|}
\hline No. & $\begin{array}{l}\text { Autor } \\
\text { (Año) }\end{array}$ & $\begin{array}{l}\text { Teoría de } \\
\text { viscosidad }\end{array}$ & $\begin{array}{l}\text { Constantes } \\
\text { del modelo }\end{array}$ & $\begin{array}{l}\text { Familias } \\
\text { orgánicas } \\
\text { evaluadas }\end{array}$ & $\begin{array}{l}\text { Ecuación } \\
\text { de estado }\end{array}$ & $\begin{array}{l}A A D \mu- \\
(M D \mu) \\
\text { Literatura }\end{array}$ & $\begin{array}{l}A A D \mu- \\
(M D \mu) \\
\text { En este Trabajo }\end{array}$ \\
\hline 1 & $\begin{array}{l}\text { Derevich } \\
(2010)\end{array}$ & $\begin{array}{l}\text { Transporte } \\
\text { de } \\
\text { momentum }\end{array}$ & 4 & n-alcanos & $\begin{array}{l}\text { Lee- } \\
\text { Kesler }\end{array}$ & $\begin{array}{l}7.00 \% \\
\text { (MD } \mu \text { no } \\
\text { reportado) }\end{array}$ & 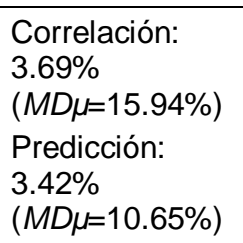 \\
\hline 2 & $\begin{array}{l}\text { Satyro y } \\
\text { Yarranton } \\
(2010)\end{array}$ & $\begin{array}{l}\text { Expansión } \\
\text { de un fluido }\end{array}$ & 16 & $\begin{array}{l}\text { n-alcanos, } \\
\text { alcanos } \\
\text { ramificados, } \\
\text { alquenos, } \\
\text { cicloalcanos y } \\
\text { aromático }\end{array}$ & $\begin{array}{l}\text { Peng- } \\
\text { Robinson }\end{array}$ & $\begin{array}{l}5.81 \% \\
\text { (MD } \mu \text { no } \\
\text { reportado) }\end{array}$ & $\begin{array}{l}\text { Correlación: } \\
5.81 \% \\
(M D \mu=20.95 \%) \\
\text { Predicción: } \\
8.25 \% \\
(M D \mu=35.68 \%)\end{array}$ \\
\hline 3 & $\begin{array}{l}\text { Novak } \\
\text { (2011) }\end{array}$ & $\begin{array}{l}\text { Teoría de } \\
\text { entropía } \\
\text { residual }\end{array}$ & 6 & n-alcanos & PC-SAFT & $\begin{array}{l}4.66 \% \\
\text { (MD } \mu \text { no } \\
\text { reportado) }\end{array}$ & $\begin{array}{l}\text { Correlación: } \\
3.69 \% \\
\text { (MD } M=15.94 \%) \\
\text { Predicción: } \\
3.42 \% \\
(M D \mu=10.65 \%)\end{array}$ \\
\hline 4 & $\begin{array}{l}\text { Bonyadi y } \\
\text { Rostami } \\
\text { (2017) }\end{array}$ & $\begin{array}{l}\text { Similitud } \\
\text { geométrica }\end{array}$ & 9 & n-alcanos & $\begin{array}{l}\text { Soave- } \\
\text { Redlich- } \\
\text { Kwong }\end{array}$ & $\begin{array}{l}7.79 \% \\
\text { (MD } \mu \text { no } \\
\text { reportado) }\end{array}$ & 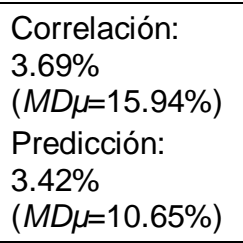 \\
\hline 5 & $\begin{array}{l}\text { Cardona } \\
\text { et al. } \\
\text { (2019a) }\end{array}$ & $\begin{array}{l}\text { Similitud } \\
\text { geométrica }\end{array}$ & 12 & $\begin{array}{l}\text { n-alcanos, n- } \\
\text { alcoholes }\end{array}$ & $\begin{array}{l}\text { Peng- } \\
\text { Robinson }\end{array}$ & $\begin{array}{l}\text { Correlación: } \\
6.74 \%(M D \mu \text { no } \\
\text { reportado) } \\
\text { Predicción: } \\
7.29 \% \\
(M D \mu=63.89 \%)\end{array}$ & 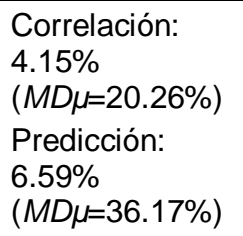 \\
\hline
\end{tabular}


Finalmente, se realiza un análisis de varianza de la $A A D \mu$ para establecer si hay una diferencia estadísticamente significativa entre las $A A D \mu$ de los modelos utilizados en la comparación de la Tabla 6. La comparación se realiza utilizando el software Statgraphics Centurion XVI (Medina-Saavedra et al., 2021). Los resultados del análisis de varianza para las $A A D \mu$ se realizan para los $\mathrm{n}$-alcanos entre metano y decano y los resultados se muestran en la Figura 5 . En esta figura se ilustra el promedio de $A A D \mu$ para cada modelo en la curva de coexistencia líquido y vapor. También se muestra un intervalo alrededor de cada media. Los intervalos mostrados se basan en el procedimiento de diferencia mínima significativa o método LSD de Fisher. Ellos se construyen de tal manera que, si dos medias son iguales, sus intervalos se solaparan el $95 \%$ de las veces. Cualquier par intervalos que no se solapen verticalmente corresponden a un par de medias las cuales tienen una diferencia estadísticamente significativa con un $\alpha=5 \%$ ( $\alpha$ es el nivel de significancia de la prueba).

De los resultados expuestos en la figura 5, los modelos de Bonyadi y Rostami (2017), Derevich (2010) y Cardona et al. (2019a) tienen iguales medias al 95\% de confianza. Por otro lado, el modelo desarrollado en este trabajo y los propuestos por Satyro y Yarranton (2010) y Novak (2011) no hay una diferencia estadísticamente significativa al 95\% de nivel de confianza. No obstante, los modelos de Satyro y Yarranton (2010) y Novak (2011) no han sido extendidos a otras familias orgánicas diferente de hidrocarburos en comparación con el desarrollado en este trabajo cuyas expresiones de generalización son simples y es aplicado a diferentes familias orgánicas de interés en la industria petroquímica y de disolventes. Los autores han verificado que se cumplen los supuestos de normalidad y homocedasticidad requeridos para la realización de esta prueba.

Finalmente, la medición de la viscosidad es afectada por diferentes factores como una inadecuada limpieza de la vidriería, burbujas o microburbujas y la presencia de agua. Además, la falta de experticia del investigador y problemas de calibración del viscosímetro constituyen elementos que generan altos valores de incertidumbre en los datos. Por estos motivos los modelos desarrollados de viscosidad tienen en cuenta este tipo de incertidumbres.

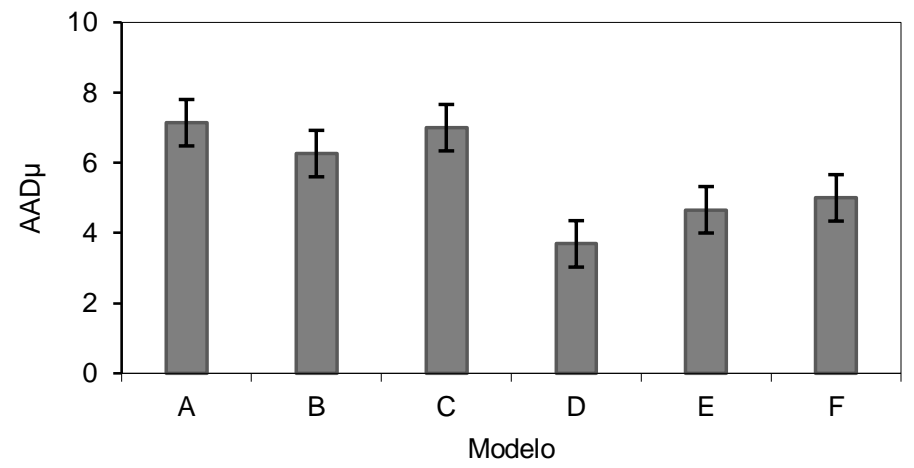

A) Bonyadi y Rostami (2017)

B) Cardona et al. (2019a)

C) Derevich (2010)

D) En este trabajo

E) Novak (2011)

F) Satyro y Yarranton (2010)

Figura 5: Comparación de medias e intervalos LSD de Fisher de la $A A D \mu$ al 95\% de confianza entre los modelos de viscosidad.

\section{CONCLUSIONES}

De acuerdo con los resultados presentados en este trabajo, se pueden extraer las siguientes conclusiones: 1) El modelo generalizado de entropía residual representa una alternativa para describir las viscosidades dinámicas de fluidos Newtonianos tanto en la fase líquida y vapor saturada de sustancias puras, no-polares y polares; 2) Las deviaciones relativas absolutas promedio durante el proceso de correlación son de $6.38 \%$ (mín. $A A D \mu=5.53 \cdot 10^{-6} \%$ y $M D \mu=26.44 \%$ ) para la fase líquida y $4.04 \%$ (mín $A A D \mu=2.82 \cdot 10^{-10}$ y $M D \mu=27.34 \%$ ) para la fase vapor; 3) Las desviaciones absolutas relativas promedio durante el proceso de predicción son de $11.36 \%$ (mín. $A A D \mu=2.49 \cdot 10^{-6} \%$ y $M D \mu=37.61 \%$ ) para la fase líquida y $5.19 \%$ (mín. $A A D \mu$ $=6.84 \cdot 10^{-9} \%$ y $M D \mu=15.10 \%$ ) para la fase vapor, siendo este proceso el valor agregado de este trabajo ya que en la literatura son pocos los modelos generalizados aplicados a diferentes familias orgánicas con predicciones aceptables; 4) Se valida el modelo generalizado frente a otros desarrollados en la literatura y se muestra que realiza predicciones de la viscosidad con bajos valores de desviación cuando es aplicado a 12 familias orgánicas y el agua (117 sustancias puras); 4) El modelo semi-empírico de viscosidad podrá ser utilizado en cálculos preliminares en el diseño de recipientes de separación líquido-líquido, líquido-vapor, torres de destilación, en simulación y optimización de procesos de transporte de petróleo y gas natural y en docencia universitaria en cursos como fenómenos de transporte y transferencia de calor y masa. 


\section{AGRADECIMIENTOS}

Los autores agradecen a la Universidad Pontificia Bolivariana y al Ministerio de Ciencia, Tecnología e Innovación de Colombia antes conocido como Departamento Administrativo de Ciencia, Tecnología e Innovación (Colciencias-Colombia), por el apoyo a través del programa Doctorados Nacionales 727 de 2015.

\section{NOTACIÓN}

Símbolos

$a, b, c$

$A, B$

$H_{1}, H_{2}$

$M_{w}$

$N$

$P$

$R$

$S$

$T$

v

Z

Abreviaciones

EoS

MS

DIPPR

$A A D$
Parámetros de la ecuación de estado descritos en la Tabla 1

Parámetros del modelo de entropía residual (adimensional)

Parámetros de Heyen

Peso molecular $\left(\mathrm{g} \cdot \mathrm{mol}^{-1}\right)$

Número total de datos

Presión (kPa)

Constante de los gases ideales

$R=8.314472 \mathrm{kPa} \cdot \mathrm{m}^{3} \cdot \mathrm{kmol}^{-1} \cdot \mathrm{K}^{-1}$

Entropía $\left(\mathrm{kJ}^{\mathrm{kmol}} \mathrm{km}^{-1} \cdot \mathrm{K}^{-1}\right)$

Temperatura (K)

Volumen molar $\left(\mathrm{m}^{3} \cdot \mathrm{kmol}^{-1}\right)$

Factor de compresibilidad (adimensional)

Ecuación de estado

Microsoft

Instituto de Diseño de Propiedades Físicas

Desviación absoluta relativa promedio

$\begin{array}{ll}\text { Abreviaciones } & \\ A D & \text { Desviación relativa } \\ M D & \begin{array}{l}\text { Máximo valor de la desviación } \\ \text { absoluta relativa }\end{array} \\ N S & \text { Número de sustancias } \\ N D & \begin{array}{l}\text { Número de datos de viscosidad } \\ \text { de cada sustancia }\end{array} \\ \text { Letras Griegas } & \\ \mu & \text { Viscosidad ( } \mu \text { P) } \\ \chi & \text { Factor polar de Halm-Stiel } \\ \omega & \text { adimensional) } \\ \text { Super/Subíndices } & \text { Factor acéntrico (adimensional) } \\ \text { cal } & \text { Calculado } \\ \text { c } & \text { Propiedad crítica } \\ \text { exp } & \text { Experimental } \\ \text { Gl } & \text { Gas ldeal } \\ \text { máx } & \text { Máximo valor } \\ \text { mín } & \text { Mínimo valor } \\ \text { res } & \text { Propiedad residual }\end{array}$

\section{REFERENCIAS}

Bell, I., y Laesecke A., Viscosity Correlations for Refrigerants and Other Working Fluids from Residual Entropy Scaling; in 16th International Refrigeration and Air Conditioning Conference at Purdue, pp 1-10 Purdue University, Indiana, USA (2016).

Bell, I.H., Probing the link between residual entropy and viscosity of molecular fluids and model potentials, https://doi.org/10.1073/pnas.1815943116, Proc. Natl. Acad. Sci., 116(10), 4070-4079 (2019).

Benabithe, Z.Z., Vanegas, D., Montoya, J.C.R., y Velásquez, J.A., Caso de estudio de la destilación etanol-agua en operación continua y discontinua y su simulación con ecuaciones cúbicas de estado y modelos de actividad, https://doi.org/10.22430/22565337.1638, TecnoLógicas, 23(49), 223-249 (2020).

Bonyadi, M. y Rostami, M., A new viscosity model based on Soave-Redlich-Kwong equation of state, https://doi.org/10.1016/j.fluid.2017.07.009, Fluid Phase Equilib., 451, 40-47 (2017).

Bloxham, J.C., Redd, M.E., Giles, N.F., Knotts, IV T.A., Wilding, W.V., Proper use of the DIPPR 801 Database for creation of models, methods, and processes, https://doi.org/10.1021/acs.jced.0c00641, J. Chem. Eng. Data, 66, 3-10 (2020).

Cardona, L.F., Forero, L.A., y Velásquez, J.A., Modelamiento de la Viscosidad con Base en una Ecuación Cúbica $\mu$ TP del Tipo Peng-Robinson, http://dx.doi.org/10.4067/S0718-07642019000400259, Inf. Tecnol., 30(4), 259-272 (2019a).

Cardona, L.F., Faúndez, C.A., Valderrama, J.O., Concepto de Similitud Geométrica para la Correlación de la Solubilidad Mutua en Mezclas Parcialmente Miscibles de Líquidos lónicos+Alcohol, http://dx.doi.org/10.4067/S071807642019000300307, Inf. Tecnol., 30(3), 307-318 (2019b).

Daubert, T.E., Evaluated equation forms for correlating thermodynamic and transport properties with temperature, https://doi.org/10.1021/ie9708687, Ind. Eng. Chem. Res., 37(8), 3260-3267 (1998).

Derevich, I.V., Thermodynamic model of viscosity of hydrocarbons and their mixtures, https://doi.org/10.1016/j.ijheatmasstransfer.2010.04.035, Int. J. Heat Mass Transf., 53(19-20), 3823-3830 (2010). 
Faúndez, C.A., y Campusano, R.A., Entrenamiento, Prueba y Predicción de la Solubilidad del Dióxido de Azufre en Líquidos lónicos utilizando Redes Neuronales Artificiales, http://dx.doi.org/10.4067/S0718-07642018000300097, Inf. Tecnol., 29(3), 97-104 (2018).

Forero, L.A. y Velásquez, J.A., Representación Simultánea del Equilibrio Líquido-Vapor, el Volumen Molar y la Entalpía de Exceso de Mezclas Complejas mediante una Ecuación de Estado tipo Peng-Robinson, http://dx.doi.org/10.4067/S0718-07642019000600021, Inf. Tecnol., 30(6), 21-34 (2019).

Galliero G., Boned, C., Fernández, J., Scaling of the viscosity of the Lennard-Jones chain fluid model, argon, and some normal alkanes, https://doi.org/10.1063/1.3553262, J. Chem. Phys., 134(6) 064505 (2011).

García, S.B., Estrada, M.R., y Silva, G.I., Correlación para el Cálculo de Viscosidades de 1-Alcoholes; Conciencia Tecnológica, 34, 66-67 (2007).

García, Y C., López, R.E.C., y Sánchez, J.G., Algoritmos genéticos en el estudio de líquidos iónicos para enfriamiento solar, https://doi.org/10.18633/biotecnia.v19i0.409, Biotecnia, 19, 38-41 (2017).

Kleiman, N., Barragán, F., y Bazúa, E., Estudio comparativo de la predicción de propiedades termodinámicas de sustancias polares con modelos recientes de ecuaciones de estado cúbicas, Rev. Mex. Ing. Quím., 1(3), 119-135 (2002).

Kontogeorgis, G.M., Folas, G.K., Thermodynamic Models for Industrial Applications: From Classical and Advanced Mixing Rules to Association Theories, Wiley (2009).

Lötgering-Lin, O. y Gross, J., Group contribution method for viscosities based on entropy scaling using the perturbed-chain polar statistical associating fluid theory, https://doi.org/10.1021/acs.iecr.5b01698, Ind. Eng. Chem. Res., 54(32), 79427952 (2015).

Márquez-Baños, E.V., Valencia-López, J.J., García-Aranda, O., y Heard, C., Determinación Computacional del Coeficiente de Transferencia de Calor en Calentadores Eléctricos de Flujo Continuo, mediante Dinámica de Fluidos Computacional, http://dx.doi.org/10.4067/S0718-07642016000500017, Inf. Tecnol., 27(5), 151-162 (2016).

Medina-Saavedra, T., Dzul-Cauich, J., Arroyo-Figueroa, G., García-Vieyra, I., Quiñones-Páramo, M., y Mexicano-Santoyo, L., Microorganismos de montaña y ensilado de maíz como probióticos en la engorda de conejos, http://dx.doi.org/10.21929/abavet2021.7, Abanico Veterinario, 11, 1-9 (2021).

Novak, L.T., Fluid viscosity-residual entropy correlation, https://doi.org/10.2202/1542-6580.2839, Int. J. Chem. React. Eng., 9(1), 1-27 (2011).

Novak, L.T., Predicting fluid viscosity of nonassociating molecules, https://doi.org/10.1021/acs.iecr.5b01526, Ind. Eng. Chem. Res., 54(21), 5830-5835 (2015).

Ortega, A.F., Pérez, A.O., y López, A.E., Modelo Semifísico de Base Fenomenológica del Proceso Continuo de Fermentación Alcohólica, http://dx.doi.org/10.4067/S0718-07642016000100004, Inf. Tecnol., 27(1), 21-32 (2016).

Páez, M.S., Alvis, A., y Arrázola, G., Efecto de la temperatura sobre la viscosidad de soluciones acuosas diluidas de cloruro de 1-butil-1-metil imidazolio [Bmim+][Cl-], http://dx.doi.org/10.4067/S0718-07642014000300021, Inf. Tecnol., 25(3), 185-190 (2014).

Poling, B.E., Prausnitz, J.M., y O'Connell, J.P., The properties of gases and liquids, Vol. 5, New York: McGraw-Hill (2001). Rosenfeld, Y., Relation between the transport coefficients and the internal entropy of simple systems, https://doi.org/10.1103/PhysRevA.15.2545, Phys. Rev. A., 15(6), 2545 (1977).

Satyro, M.A. y Yarranton, H.W., Expanded fluid-based viscosity correlation for hydrocarbons using an equation of state, https://doi.org/10.1016/j.fluid.2010.06.023, Fluid Phase Equilib., 298(1), 1-11 (2010).

Valderrama, J.O., The state of the cubic equations of state, https://doi.org/10.1021/ie020447b, Ind. Eng. Chem. Res., 42(8), 1603-1618 (2003).

Valderrama, J.O., Muñoz, J.M., y Rojas, R.E., Viscosity of ionic liquids using the concept of mass connectivity and artificial neural networks, https://doi.org/10.1007/s11814-010-0512-0, Korean J. Chem. Eng., 28(6), 1451-1457 (2011).

Valderrama, J.O. y Rojas, R.E., Avances en la predicción de propiedades físicas, físico-químicas y de transporte de líquidos iónicos, http://dx.doi.org/10.4067/S0718-07642009000400017, Inf. Tecnol., 20(4), 149-160 (2009).

Valderrama, J.O., Cardona, L.F., y Rojas, R.E., Correlation and prediction of ionic liquid viscosity using Valderrama-PatelTeja cubic equation of state and the geometric similitude concept. Part l: pure ionic liquids, https://doi.org/10.1016/j.fluid.2019.04.031, Fluid Phase Equilib., 497, 164-177 (2019). 